OPEN ACCESS

Edited by:

Christopher J. A. Duncan, Newcastle University, United Kingdom

Reviewed by: Johan Van Weyenbergh,

KU Leuven, Belgium

Anthony Jaworowski,

RMIT University, Australia

${ }^{*}$ Correspondence: Ana Copaescu ana.copaescu@gmail.com

${ }^{+}$These authors share first authorship

${ }^{\ddagger}$ These authors share senior authorship

Specialty section: This article was submitted to Viral Immunology, a section of the journal

Frontiers in Immunology

Received: 25 December 2020 Accepted: 25 February 2021

Published: 17 March 2021

Citation:

Copaescu A, James F, Mouhtouris E, Vogrin S, Smibert OC, Gordon CL,

Drewett G, Holmes NE and

Trubiano JA (2021) The Role of Immunological and Clinical Biomarkers to Predict Clinical COVID-19 Severity and Response to Therapy $-A$ Prospective Longitudinal Study.

Front. Immunol. 12:646095. doi: 10.3389/fimmu.2021.646095

\section{The Role of Immunological and Clinical Biomarkers to Predict Clinical COVID-19 Severity and Response to Therapy-A Prospective Longitudinal Study}

\author{
Ana Copaescu ${ }^{1,2,3{ }^{*}+}$, Fiona James ${ }^{1 \dagger}$, Effie Mouhtouris ${ }^{1}$, Sara Vogrin ${ }^{4}$, Olivia C. Smibert ${ }^{5}$, \\ Claire L. Gordon ${ }^{5,6}$, George Drewett ${ }^{5}$, Natasha E. Holmes ${ }^{1,5,7 \neq}$ \\ and Jason A. Trubiano ${ }^{1,5,8,9 \neq}$ \\ ${ }^{1}$ Centre for Antibiotic Allergy and Research, Department of Infectious Diseases, Austin Health, Heidelberg, VIC, Australia, \\ ${ }^{2}$ Clinical Immunology and Allergy, Department of Medicine, McGill University Health Center, Montréal, QC, Canada, \\ ${ }^{3}$ The Research Institute of the McGill University Health Centre, McGill University, Montreal, QC, Canada, ${ }^{4}$ Department of \\ Medicine, St Vincent's Hospital, University of Melbourne, Fitzroy, VIC, Australia, ${ }^{5}$ Department of Medicine (Austin Health), \\ The University of Melbourne, Heidelberg, VIC, Australia, ${ }^{6}$ Department of Microbiology and Immunology, The University of \\ Melbourne, Parkville, VIC, Australia, ${ }^{7}$ Department of Critical Care, Melbourne Medical School, The University of Melbourne, \\ Parkville, VIC, Australia, ${ }^{8}$ Department of Oncology, Sir Peter MacCallum Cancer Centre, The University of Melbourne, Parkville, \\ VIC, Australia, ${ }^{9}$ The National Centre for Infections in Cancer, Peter MacCallum Cancer Centre, Parkville, VIC, Australia
}

Background: The association of pro-inflammatory markers such as interleukin-6 (IL-6) and other biomarkers with severe coronavirus disease 2019 (COVID-19) is of increasing interest, however their kinetics, response to current COVID-related treatments, association with disease severity and comparison with other disease states associated with potential cytokine storm (CS) such as Staphylococcus aureus bacteraemia (SAB) are ill-defined.

Methods: A cohort of 55 hospitalized SARS-CoV-2 positive patients was prospectively recruited - blood sampling was performed at baseline, post-treatment and hospital discharge. Serum IL-6, C-reactive protein (CRP) and other laboratory investigations were compared between treatment groups and across timepoints. Acute serum IL-6 and CRP levels were then compared to those with suspected COVID-19 (SCOVID) and age and sex matched patients with SAB and patients hospitalized for any non-infectious condition (NIC).

Results: IL-6 was elevated at admission in the SARS-CoV-2 cohort but at lower levels compared to matched SAB patients. Median (IQR) IL-6 at admission was $73.89 \mathrm{pg} / \mathrm{mL}$ (30.9, 126.39) in SARS-CoV-2 compared to $92.76 \mathrm{pg} / \mathrm{mL}(21.75,246.55)$ in SAB $(\mathrm{p}=0.017) ; 12.50 \mathrm{pg} / \mathrm{mL}(3.06,35.77)$ in patients with $\mathrm{NIC}$; and $95.51 \mathrm{pg} / \mathrm{mL}(52.17$, 756.67) in SCOVID. Median IL-6 and CRP levels decreased between admission and discharge timepoints. This reduction was amplified in patients treated with remdesivir and/ 
or dexamethasone. CRP and bedside vital signs were the strongest predictors of COVID19 severity.

Conclusions: Knowledge of the kinetics of IL-6 did not offer enhanced predictive value for disease severity in COVID-19 over common investigations such as CRP and vital signs.

Keywords: SARS-CoV-2, interleukin-6, C-reactive protein, cytokine storm, Staphylococcus aureus bacteraemia, sepsis, acute respiratory distress syndrome

\section{INTRODUCTION}

There has been increasing interest surrounding the function of interleukin-6 (IL-6) and other laboratory markers in severe acute respiratory syndrome coronavirus 2 (SARS-CoV-2) infection, including the role in predicting disease severity, monitoring response to therapy, and similarities with other cytokine storm (CS) disease states (1-4). Heterogeneity in SARS-CoV-2 study design and definitions of disease severity have limited advances in understanding the clinical implications for IL- 6 and other inflammatory and clinical makers in coronavirus disease 2019 (COVID-19) (4). Further, IL-6 kinetics in SARS-CoV-2 and comparisons with other infective syndromes with CS, such as bacterial sepsis, have not been extensively undertaken.

\section{METHODS}

\section{Participants and Setting}

Adults aged $\geq 18$ years hospitalized with suspected or confirmed SARS-CoV-2, no known hypersensitivity to tocilizumab and no active pulmonary tuberculosis, were enrolled sequentially in this single-center prospective cohort study between May $15^{\text {th }} 2020$ and August $21^{\text {st }} 2020$ at Austin Health, Melbourne, Australia.

\section{Definitions}

Severe disease was defined as requirement for supplemental oxygen for $\geq 24$ hours and a Sp02 $\leq 94 \%$ on room air and/or admission to intensive care unit (ICU), adapted from the National Institutes of Health (NIH) criteria for disease severity (5). Treatment for COVID-19 was as per hospital approved treatment protocol. Patients that required oxygen received dexamethasone, remdesivir was utilized in those that had oxygenation $<94 \%$ and were early in disease and tocilizumab was only considered on a case by case basis in intubated patients with evidence of cytokine storm (4) and no occult sepsis (negative procalcitonin). A 5-day course of remdesivir and/or dexamethasone for up to 10 days was administered to patients with severe disease starting on the $23^{\text {rd }}$ of June 2020.

\footnotetext{
Abbreviations: ARDS, acute respiratory distress syndrome; CS, cytokine storm; CRP, C-reactive protein; COVID-19, coronavirus disease 2019; ICU, intensive care unit; IL-6, interleukin-6; NIC, non-infectious condition; SAB, Staphylococcus aureus bacteraemia; SCOVID, suspected COVID-19; SARS-CoV-2, severe acute respiratory syndrome coronavirus 2 .
}

\section{Objectives}

The primary objective of the study was to describe the kinetics of IL-6 and other biomarkers during SARS-CoV-2 infection. Secondary objectives were to describe the association of these markers with (1) disease severity (defined as ICU admission, oxygen therapy or a composite of both), (2) treatment with dexamethasone and/or remdesivir, and (3) other disease states associated with CS such as Staphylococcus aureus bacteraemia (SAB).

\section{Data Collection and Cohorts}

Standard baseline demographic and clinical characteristics, laboratory parameters (including C-reactive protein (CRP), lymphocyte count, ferritin, lactate dehydrogenase (LDH) and D-dimer) and COVID-related treatment data (requirement for oxygen therapy and/or intubation, drug therapy with dexamethasone and/or remdesivir) were gathered. Serum samples were collected at four timepoints: (1) hospital admission, (2) 24 to 48 hours post dexamethasone and/or remdesivir, (3) 7 to 14 days post-treatment, and (4) discharge (Figure 1).

We compared the SARS-CoV-2 group with three distinct inpatient cohorts with potentially varied cytokine storm disease states: (1) patients with SAB; (2) patients hospitalized for any non-infectious conditions (NIC); and (3) patients with suspected COVID-19 (SCOVID) concurrently recruited with the SARSCoV-2 patients from Austin Hospital. The patients with SCOVID had a minimum of 2 negative COVID-19 tests upon hospital admission as well as repeated testing depending on their clinical evolution. The SARS-CoV-2 patients were age and sex matched with previously described SAB (6) and NIC (7) cohorts (Figure 1). No other demographic information was available for the patients recruited in theses cohorts. The serum samples for the SAB and NIC cohorts were stored since their initial recruitment in accordance with well-known laboratory storing procedures at $-80^{\circ} \mathrm{C}$ ensuring adequate back-up capacity for the freezers under the supervision of trained personnel and with ongoing alarm systems designed to monitor the temperature. Serum IL-6 from each patient cohort was quantified using an enzyme-linked immunosorbent assay (ELISA) assay (Crux Biolabs $^{\circledR}$, Australia) following manufacturers' instructions (8) and read at a wavelength of $450 \mathrm{~nm}$ with a FLUOstar Optima plate reader (BMG labtech ${ }^{\circledR}$ ).

\section{Statistical Analysis}

Categorical variables are reported as counts and percentages; continuous variables as medians (interquartile range, IQR). IL-6 


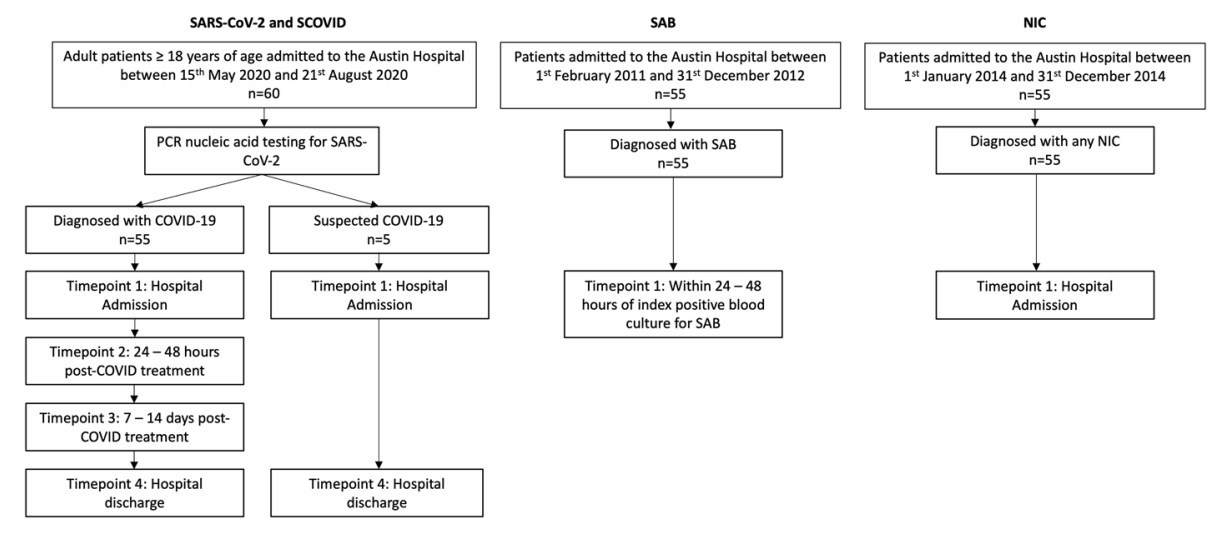

FIGURE 1 | Study design outlining patient cohorts and time points for sample collection. SAB and NIC cohorts are age and sex matched with the SARS-CoV-2 cohort. PCR, polymerase chain reaction; NIC, non-infectious condition; SAB, Staphylococcus aureus bacteraemia; SARS-CoV-2, severe acute respiratory syndrome coronavirus.

TABLE 1 | Patient characteristics of SARS-CoV-2 positive cohort.

\section{Characteristics}

\section{COVID (n = 55)}

\begin{tabular}{ccc}
\hline All patients $(\mathbf{n}=\mathbf{5 5})$ & ICU Admission $(\mathbf{n}=\mathbf{1 5})$ & Supplemental $\mathbf{O}_{\mathbf{2}} \mathbf{( n = 2 5 )} \boldsymbol{\Psi}$ \\
\hline $58(40 ; 70)$ & $59(50 ; 69)$ & $66(52 ; 71)$ \\
$31: 24$ & $10: 5$ & $17: 8$ \\
ATSI $(1 ; 1.8 \%)$ & African $(3 ; 20 \%)$ & African $(2 ; 8 \%)$ \\
African $(4 ; 7.3 \%)$ & Caucasian $(8 ; 53.3 \%)$ & Caucasian $(17 ; 68 \%)$ \\
Caucasian $(31 ; 56.4 \%)$ & Indo-Asian $(1 ; 6.7 \%)$ & East Asian $(1 ; 4 \%)$ \\
East Asian $(4 ; 7.3 \%)$ & Other $(1 ; 6.7 \%)$ & Indo-Asian $(2 ; 4 \%)$ \\
Indo-Asian $(2 ; 3.6 \%)$ & Unknown $(2 ; 13.3 \%)$ & Other $(1 ; 4 \%)$ \\
Other $(1 ; 1.8 \%)$ & & Unknown $(3 ; 12 \%)$
\end{tabular}

Smoking status

(no./total no.; \%)

Unknown $(12 ; 21.8 \%)$

Smoker (4/50; 8\%)

Ex-smoker (6/50;12\%)

Non-smoker (40/50; 80\%)

\section{Comorbidities (no.; \%)}

Hypertension

Cardiac disease

Chronic respiratory disease

Chronic renal or liver disease

Diabetes

Immunosuppression \&

Pregnancy

ACEI/ARB use

Clinical characteristics

Charlson comorbidity index

(median, IQR)

COVID-MATCH65 Score $\Phi$

(median, IQR)

Latency presentation recruitment $\nabla$ (days), (median, IQR)

Length of hospital stay (days)

(median, IQR)

Death (no.; \%)
$20 ; 36.4 \%$
$7 ; 12.7 \%$
$13 ; 23.6 \%$
$1 ; 1.8 \%$
$15 ; 27.3 \%$
$5 ; 9.1 \%$
$1 ; 1.8 \%$
$10 ; 18.2 \%$

$$
1(0 ; 3)
$$

$3.5(2.5 ; 5)$

$7(5 ; 10)$

$6(3 ; 13)$

$2 ; 3.6 \%$
Smoker (1/13; 7.7\%)

Ex-smoker (1/13; 7.7\%)
Non-smoker (11/13; 84.6\%)

Smoker (1/21; 4.8\%)

Ex-smoker (4/21; 19\%)

Non-smoker (16/21; 76.2\%)

\begin{tabular}{cc}
$6 ; 40 \%$ & $14 ; 56 \%$ \\
$2 ; 13.3 \%$ & $5 ; 20 \%$ \\
$5 ; 33.3 \%$ & $9 ; 36 \%$ \\
$0 ; 0 \%$ & $0 ; 0 \%$ \\
$5 ; 33.3 \%$ & $10 ; 40 \%$ \\
$2 ; 13.3 \%$ & $3 ; 12 \%$ \\
$0 ; 0 \%$ & $0 ; 0 \%$ \\
$2 ; 13.3 \%$ & $7 ; 28 \%$ \\
& \\
$1(1 ; 2)$ & $2(1 ; 3)$ \\
$4(3.5 ; 5)$ & \\
$7(5 ; 10)$ & $4.5(3.5 ; 5)$ \\
$17(9 ; 27)$ & $7(5 ; 9)$ \\
$0 ; 0 \%$ & $13(6 ; 21)$ \\
\hline
\end{tabular}

ACEl, angiotensin-converting-enzyme inhibitors; ARB, angiotensin II receptor blockers; ATSI, Aboriginal or Torres Strait Islander; ICU, intensive care unit; SARS-CoV-2, severe acute respiratory syndrome coronavirus 2.

* The immunosuppression category includes patients that are known for any of the following conditions: transplant recipient, hematological or oncological malignancy (in the last 5 years), corticosteroid use of more than $10 \mathrm{mg}$ prednisolone equivalent per day, connective tissue or autoimmune condition and acquired immunodeficiency syndrome.

$\Psi$ Patients that required supplemental $\mathrm{O}_{2}$ continuously for more than 24 hours during their admission.

- The Charlson comorbidity index is age-adjusted.

Ф COVID-MATCH65 Score is a clinical decision rule internally derived that has a high sensitivity (92.6\%) and NPV (99.5\%) for SARS-CoV-2 and can be used to aid COVID-19 risk assessment and resource allocation for SARS-CoV-2 diagnostics. The resulting score ranges from 1 to 6.5 points with score $\leq 1$ representing low risk for a positive test (9).

$\nabla$ Time from symptoms presentation and study recruitment (days). 
levels across time in the treatment groups were compared using mixed effects linear regression, while linear regression was used to compare between treatment groups. The outcome was logtransformed and the results reported as exponentiated regression coefficients $(95 \% \mathrm{CI})$. Characteristics of matched cohorts (COVID, SAB and NIC) were compared using sign-rank test and McNemar test. IL-6 levels between cohorts (SAB vs COVID and NIC vs COVID) were compared using paired t-test. Univariable logistic regression was used for evaluation of demographic, clinical and laboratory variables with supplemental oxygen requirement. Results were expressed as odds ratios (95\% CI). Competing risk time to event analysis was performed to measure the association of IL-6 and CRP with oxygen supplementation. The measured treatment outcomes were admission to ICU, oxygen therapy or a composite of both. Discharge was taken as competing risk and IL-6/CRP were separately entered as admission values and as timevarying covariates. Results were reported as sub-hazard ratios

TABLE 2 | Clinical, laboratory characteristics and treatment information for SARS-CoV-2 positive patients.

\begin{tabular}{|c|c|c|c|}
\hline & All patients $(n=55)$ & ICU Admission ( $n=15)$ & Supplemental $\mathrm{O}_{2}(\mathrm{n}=25) \Psi$ \\
\hline \multicolumn{4}{|c|}{ Patient reported clinical symptoms at baseline (no.; \%) } \\
\hline Fever $>38^{\circ} \mathrm{C}$ & $26 ; 47.3 \%$ & $9 ; 60.0 \%$ & $14 ; 56.0 \%$ \\
\hline Malaise/myalgia & $32 ; 58.2 \%$ & $8 ; 53.3 \%$ & $14 ; 56.0 \%$ \\
\hline Dyspnea & $42 ; 76.4 \%$ & $14 ; 93.3 \%$ & $22 ; 88.0 \%$ \\
\hline Cough & $36 ; 65.5 \%$ & $9 ; 60.0 \%$ & $15 ; 60.0 \%$ \\
\hline Coryza & $9 ; 16.4 \%$ & $2 ; 13.3 \%$ & $1 ; 4.0 \%$ \\
\hline Sore throat & $16 ; 29.1 \%$ & $2 ; 13.3 \%$ & $2 ; 8.0 \%$ \\
\hline Diarrhea & $16 ; 29.1 \%$ & $4 ; 26.7 \%$ & $7 ; 28.0 \%$ \\
\hline \multirow[t]{4}{*}{ Other } & Headache (3; 0.05\%) & Headache (0; 0\%) & Headache (0; 0\%) \\
\hline & $\begin{array}{c}\text { Nausea and Vomiting } \\
(2 ; 0.04 \%)\end{array}$ & $\begin{array}{l}\text { Nausea and Vomiting } \\
\qquad(0 ; 0 \%)\end{array}$ & $\begin{array}{l}\text { Nausea and Vomiting } \\
\qquad(1 ; 0.04 \%)\end{array}$ \\
\hline & Pleuritic chest pain & Pleuritic chest pain & Pleuritic chest pain \\
\hline & $(4 ; 0.07 \%)$ & $(0 ; 0 \%)$ & $(1 ; 0.04 \%)$ \\
\hline \multicolumn{4}{|c|}{ Vital signs - baseline at hospital admission (median, IQR) } \\
\hline Temperature $\left({ }^{\circ} \mathrm{C}\right)$ & $37.8(36.7 ; 38.5)$ & $38.1(37.1 ; 38.7)$ & $38.3(37.3 ; 38.8)$ \\
\hline Respiratory rate & $22(20 ; 30)$ & $35(22 ; 38)$ & $28(22 ; 35)$ \\
\hline Oxygen saturation (\%) & $95(92 ; 98)$ & $94(90 ; 96)$ & $92(90 ; 94)$ \\
\hline Pulse rate & $96(88 ; 106)$ & $100(92 ; 119)$ & $100(88 ; 115)$ \\
\hline \multirow[t]{2}{*}{ Blood pressure (mmHg) } & $118 / 72$ & $118 / 78$ & $120 / 69$ \\
\hline & $(107 / 66 ; 130 / 81)$ & $(100 / 60 ; 130 / 85)$ & $(103 / 64 ; 130 / 80)$ \\
\hline \multicolumn{4}{|c|}{ Laboratory Data - baseline (median, IQR) } \\
\hline $\operatorname{WCC}\left(\times 10^{9} / \mathrm{L}\right)$ & $6(4.4 ; 8)$ & $7.2(4.4 ; 8)$ & $7.1(4.3 ; 8)$ \\
\hline Lymphocytes (x10/L) & $0.8(0.7 ; 1.1)$ & $0.7(0.5 ; 0.8)$ & $0.8(0.6 ; 1)$ \\
\hline Neutrophils $\left(\times 10^{9} / \mathrm{L}\right)$ & $4.3(2.9 ; 6)$ & $5.8(2.9 ; 6.6)$ & $5.1(2.9 ; 6.2)$ \\
\hline Eosinophils (x109/L) & $0(0 ; 0)$ & $0(0 ; 0)$ & $0(0 ; 0)$ \\
\hline Hemoglobin (g/L) & $135(123 ; 146)$ & $139(125: 145)$ & $139(127 ; 145)$ \\
\hline Platelet count $\left(\times 10^{9} / \mathrm{L}\right)$ & $202(171 ; 257)$ & $194(161 ; 253)$ & $194(161 ; 253)$ \\
\hline Creatinine $(\mu \mathrm{mol} / \mathrm{L})$ & $74(60 ; 94)$ & $82(64 ; 95)$ & $82(69 ; 104)$ \\
\hline Estimated GFR & $90(71 ; 90)$ & $86(71 ; 90)$ & $79(64 ; 90)$ \\
\hline Sodium (mmol/L) & $139(136 ; 141)$ & $138(135 ; 141)$ & $139(136 ; 141)$ \\
\hline Potassium (mmol/L) & $4.1(3.9 ; 4.4) ; \mathrm{N}=53$ & $4(4 ; 4.4) ; N=13$ & $4.1(4 ; 4.5) ; N=23$ \\
\hline Bicarbonate (mmol/L) & $25(22 ; 27)$ & $25(24 ; 27)$ & $25(24 ; 27)$ \\
\hline IL-6 (pg/ml) & 73.9 (30.9;126.39) & $56.6(21.3 ; 108.3)$ & $73.9(31.1 ; 123.2)$ \\
\hline $\mathrm{CRP}(\mathrm{mg} / \mathrm{L})$ & 65 (19.4; 135); N=53 & 135 (49.1; 223); N=15 & 113 (44.9; 196.5); N=24 \\
\hline Ferritin $(\mu \mathrm{g} / \mathrm{L})$ & $438(167 ; 864) ; N=50$ & 1,084 (490; 1,570); N=15 & $645.5(209 ; 1,311) ; \mathrm{N}=24$ \\
\hline D-dimer (mg/L) & $635(473 ; 972) ; N=53$ & $1,394(738 ; 2,505) ; \mathrm{N}=15$ & $969(680 ; 1,542) ; \mathrm{N}=25$ \\
\hline LDH (U/L) & 278 (236; 366); N=33 & $402.5(326 ; 525) ; \mathrm{N}=10$ & $363(267 ; 525) ; \mathrm{N}=15$ \\
\hline Bilirubin ( $\mu \mathrm{mol} / \mathrm{L})$ & $8(6.5 ; 13) ; N=48$ & $10(7 ; 15) ; N=13$ & 8 (6;15); N=22 \\
\hline ALT (U/L) & $28(21 ; 50) ; N=50$ & $37(21 ; 49) ; N=14$ & $28.5(20 ; 47) ; N=24$ \\
\hline AST (U/L) & $65(55 ; 85) ; N=13$ & $66(55 ; 189) ; N=3$ & $62.5(55 ; 66) ; \mathrm{N}=6$ \\
\hline GGT (U/L) & $42(25 ; 82)$ & $63(40 ; 109)$ & $55(38 ; 84)$ \\
\hline Albumin (g/L) & $35(31 ; 37) ; N=53$ & $31(26 ; 36) ; N=15$ & 32.5 (30; 36); N=24 \\
\hline \multicolumn{4}{|l|}{ Treatment (no.; \%) } \\
\hline Mechanical ventilation & $5(9.1 \%)$ & 5 (33.3\%) & $5(20 \%)$ \\
\hline Dexamethasone & $28(50.9 \%)$ & $12(80 \%)$ & 22 (88\%) \\
\hline Remdesivir and dexamethasone & $15(27.3 \%)$ & $8(53.5 \%)$ & $13(52 \%)$ \\
\hline Intravenous antibiotics & $35(63.6 \%)$ & 15 (100\%) & 22 (88\%) \\
\hline Antifungals & $3(5.5 \%)$ & $3(20 \%)$ & $3(12 \%)$ \\
\hline
\end{tabular}

ALT, alanine aminotransferase; AST, aspartate transaminase; CRP, C-Reactive protein; GFR, glomerular filtration rate; GGT, gamma-glutamyl transferase; ICU, intensive care unit; IL-6, Interleukin-6; $L D H$, lactate dehydrogenase; SARS-CoV-2, severe acute respiratory syndrome coronavirus 2; SpO2, oxygen saturation; WCC, white cell count. $\Psi$ Patients that required supplemental $\mathrm{O}_{2}$ continuously for more than 24 hours during their admission.

- Estimated GFR was calculated using Chronic Kidney Disease Epidemiology Collaboration (CKD-EPI), units: ml/min/1.73. 
(SHRs) with 95\% CI. Stata/IC 16.1 was used for all analysis. The study was approved by the local human research ethics committee (Ref HREC/63201/Austin-20). All patients, their legal representatives or their next of kin provided informed consent for this study.

\section{RESULTS}

\section{Cohort Characteristics}

The baseline demographics and clinical characteristics for SARSCoV-2 positive cohort $(n=55)$ are described in Table 1 . The laboratory values and treatment information are listed in Table 2. Twenty-five $(45 \%)$ of the patients required continuous supplemental oxygen for $\geq 24$ hours after $\mathrm{SpO}_{2}$ fell below $94 \%$ on room air and $15(27 \%)$ were admitted to ICU. Two patients died while in hospital. There were 28 patients who received dexamethasone $(50.9 \%)$; 15 who received remdesivir $(27.3 \%)$ and $15(27.3 \%)$ who received both.

\section{IL-6 and Biomarker Kinetics in SARS-CoV- 2-Infected Patients}

The kinetics of exploratory biomarkers over time, stratified by treatment group (dexamethasone, dexamethasone and remdesivir, no treatment) can be visualized in Figure 2. IL-6 and CRP values decreased with time from admission across all groups. Ferritin, LDH and D-dimer were marginally decreased from admission to discharge but increased post-treatment for the small numbers of patients in this group and lymphocytes were slightly increased from admission to discharge (Figure 2). At
A

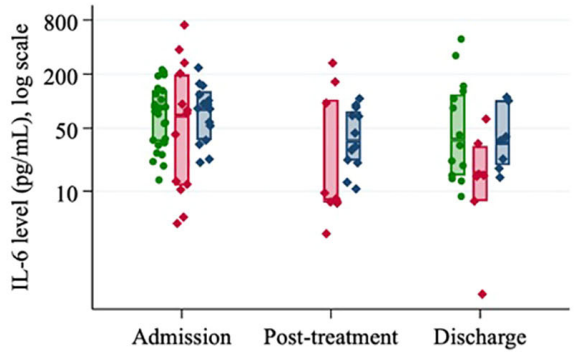

C
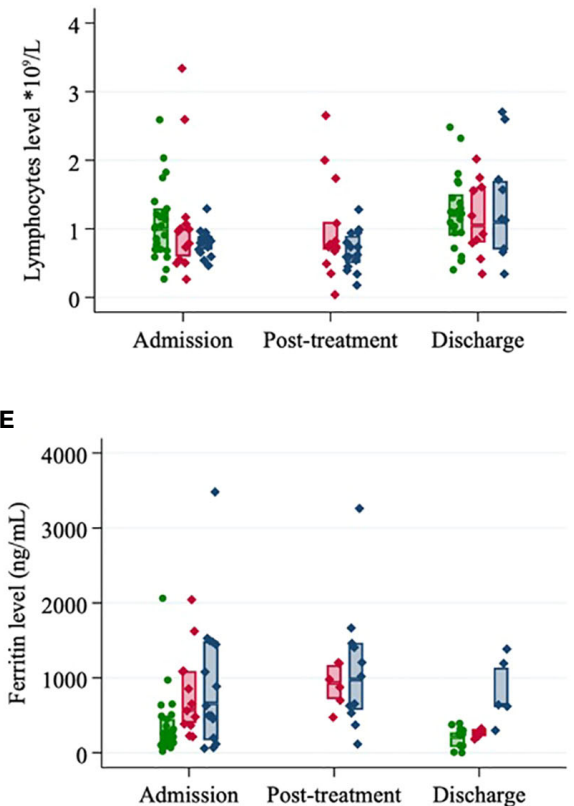

B

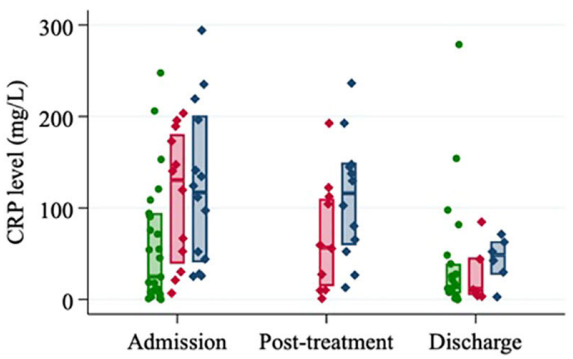

D
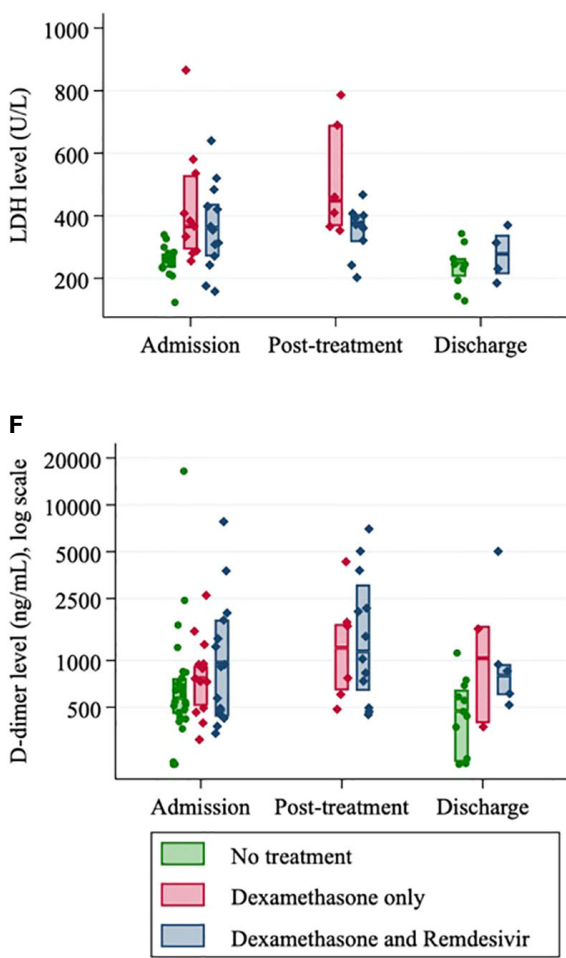

FIGURE 2 | Laboratory data for SARS-CoV-2 positive patients ( $n=55)$ according to their treatment regimen and at different admission timepoints (median, IQR): (A) Interleukin-6; (B) C-Reactive protein; (C) Lymphocytes; (D) Lactate dehydrogenase (LDH); (E) Ferritin level and (F) D-dimer. 
admission, there was no difference in serum IL-6 levels between those who received treatment and those who did not (Table 3). Post-treatment levels of serum IL-6 halved in the remdesivir and dexamethasone treatment group $(\mathrm{p}=0.023)$ (Table 3). At discharge, IL-6 was 48\% lower than at admission for those who received remdesivir and dexamethasone $(p=0.059)$ and $83 \%$ lower in the dexamethasone only group $(\mathrm{p}=0.003)$ (Table 3).

\section{IL-6 in SARS-CoV-2 Infected Patients Compared With Other Disease States}

Serum IL-6 at hospital admission in SARS-CoV-2 positive patients was compared with the SCOVID, SAB and NIC groups (Figure 3). IL-6 values are demonstrated in Supplementary Tables 1 and 2. A mean difference of $72.7 \mathrm{pg} /$ $\mathrm{ml}(95 \% \mathrm{CI} ; 40.0,105.3)$ was found between the NIC and SARSCoV-2 groups $(\mathrm{p}<0.001)$. Mean IL-6 was elevated in the SAB group by $57.8 \mathrm{pg} / \mathrm{ml}(95 \% \mathrm{CI} ; 0.3,115.2)$ over the SARS-CoV-2 group $(\mathrm{p}=0.049)$. The univariable associations with ICU admission for SAB and SARS-CoV-2 positive cohorts are illustrated in Table 4. Although CRP was associated with ICU admission in both cohorts, IL-6 was associated with ICU admission only in the SAB cohort.

\section{Association Between IL-6 and Other Biomarkers With Clinical Outcomes in SARS-CoV-2 Infected Patients}

No association between elevated baseline IL- 6 and either requirement for oxygen therapy, ICU admission or composite outcomes was found on univariable analysis (Supplementary Tables 3 and 4). An increased CRP of $10 \mathrm{mg} / \mathrm{L}$ increased odds of oxygen therapy requirement by $13 \%(\mathrm{p}=0.006)$, ICU admission by $1 \%(\mathrm{p}=0.014)$ and the composite outcome by $1 \%(\mathrm{p}=0.003)$.

TABLE 3 | Impact of COVID-19 treatment on IL-6 values.

\begin{tabular}{|c|c|c|}
\hline & OR $(95 \% \mathrm{Cl})$ & $p$ - value \\
\hline \multicolumn{3}{|l|}{ Effect of time } \\
\hline \multicolumn{3}{|l|}{ No treatment } \\
\hline Discharge vs admission & $0.76(0.47,1.22)$ & 0.258 \\
\hline \multicolumn{3}{|l|}{ Remdesivir and dexamethasone } \\
\hline Post treatment vs admission & $0.52(0.29,0.91)$ & 0.023 \\
\hline Discharge vs admission & $0.52(0.27,1.03)$ & 0.059 \\
\hline \multicolumn{3}{|l|}{ Dexamethasone } \\
\hline Post treatment vs admission & $0.54(0.18,1.59)$ & 0.266 \\
\hline Discharge vs admission & $0.17(0.05,0.55)$ & 0.003 \\
\hline \multicolumn{3}{|l|}{ Comparison between groups } \\
\hline \multicolumn{3}{|l|}{ Admission } \\
\hline Treatment vs no treatment & $0.91(0.52,1.62)$ & 0.756 \\
\hline $\begin{array}{l}\text { Remdesivir (+dexamethasone vs } \\
\text { dexamethasone }\end{array}$ & $1.45(0.54,3.86)$ & 0.442 \\
\hline \multicolumn{3}{|l|}{ Post-treatment } \\
\hline $\begin{array}{l}\text { Remdesivir (+dexamethasone) vs } \\
\text { dexamethasone }\end{array}$ & $1.91(0.62,5.84)$ & 0.242 \\
\hline \multicolumn{3}{|l|}{ Discharge } \\
\hline Treatment vs no treatment & $0.45(0.17,1.20)$ & 0.106 \\
\hline Remdesivir (+dexamethasone) vs & $3.03(0.79,11.71)$ & 0.099 \\
\hline
\end{tabular}

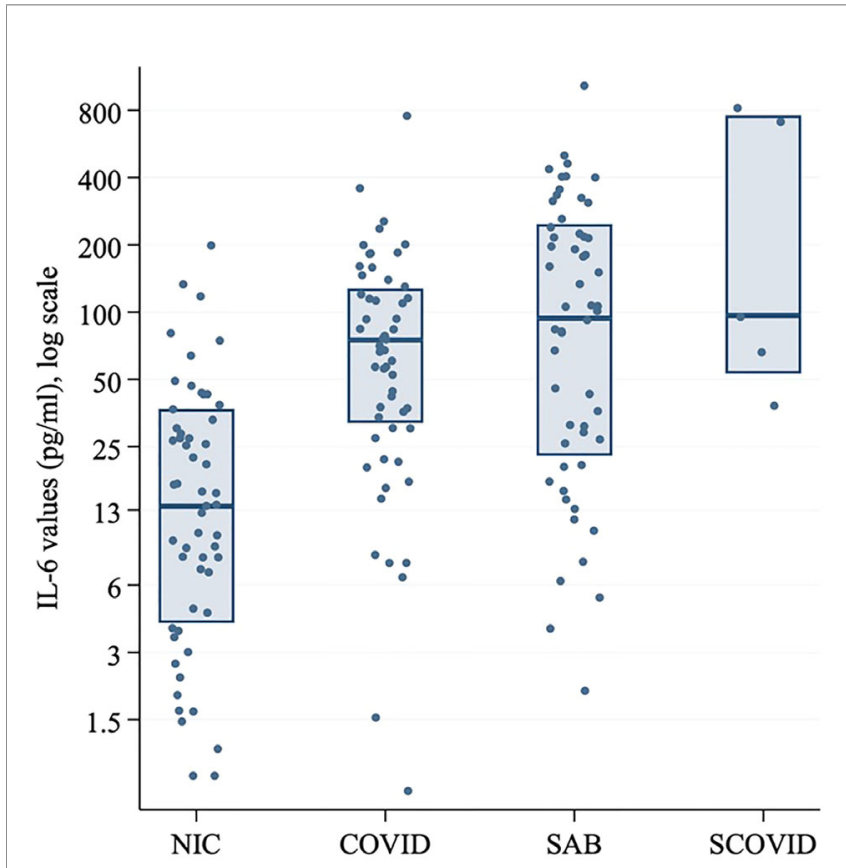

FIGURE 3 | Log-transformed IL-6 values (pg/ml) for SARS-CoV-2 positive patients at baseline, SCOVID, SAB and a cohort of hospitalized patients for any NIC. The SARS-CoV-2 positive patients were age and gender matched with patients from SAB and NIC cohorts. CRP, C-Reactive protein; COVID, coronavirus disease; IL-6, Interleukin-6; LDH, Lactic acid dehydrogenase; NIC, non-infectious condition; SAB, Staphylococcus aureus bacteraemia; SARS-CoV-2, severe acute respiratory syndrome coronavirus 2; SCOVID, suspected COVID-19.

Using time to event analysis, IL-6 did not appear to be associated with requirement for oxygen therapy, ICU admission or a composite endpoint of both outcomes (Table 5). In contrast, increased CRP was still associated with oxygen therapy and the composite endpoint, even when adjusted for predictors of the outcome identified on logistic regression. After adjustment for respiratory rate and $\mathrm{SpO}_{2}$, an increase of $10 \mathrm{mg} / \mathrm{mL}$ in CRP was associated with a $5 \%$ increased risk of requirement for oxygen therapy $(\mathrm{p}=0.013)$ and a $5 \%$ increased risk of the composite outcome $(\mathrm{p}=0.025)$.

\section{DISCUSSION}

This study presents unique prospectively data with multiple time-point sampling, assessing IL-6 and other inflammatory and clinical biomarkers in response to an antiviral (i.e. remdesivir) and an immunosuppressant (i.e. dexamethasone) treatment - informing strategies to predicting clinical severity and response to therapy. IL- 6 is secreted by a plethora of immune and stromal cells and exerts effects on a similarly broad array of cellular targets translating into functional pleiotropy including the synthesis of acute phase proteins in the liver, such as C-reactive protein (CRP), a surrogate for IL-6 $(10,11)$. CRP is 
TABLE 4A | Admission characteristics and univariable model for association of ICU admission in SARS-CoV-2, Staphylococcus aureus bacteremia (SAB), SARS-CoV-2 positive and suspected COVID-19 (SCOVID) cohorts.

\begin{tabular}{|c|c|c|c|c|c|}
\hline Factors & SAB & SARS-CoV-2 positive & p-value $\Psi$ & NIC & SCOVID \\
\hline $\mathrm{N}$ & 55 & 55 & & 55 & 5 \\
\hline Female, no. (\%) & 25 (45\%) & 24 (44\%) & 0.564 & $25(45 \%)$ & $2(40 \%)$ \\
\hline Age, median (IQR) & $58(41,70)$ & $58(40,70)$ & 0.309 & $58(41,70)$ & $68(54,75)$ \\
\hline $\mathrm{CCl}$, median (IQR) & $2(0,3)$ & $1(0,3)$ & 0.344 & $\mathrm{n} / \mathrm{a}$ & $\mathrm{n} / \mathrm{a}$ \\
\hline ICU admission & $14(25 \%)$ & $15(27 \%)$ & 0.835 & $\mathrm{n} / \mathrm{a}$ & $0(0 \%)$ \\
\hline WCC, median (IQR) & $10(6.8,15.6)$ & $6(4.4,8)$ & $<0.001$ & $\mathrm{n} / \mathrm{a}$ & $7.6(1.6,10.9)$ \\
\hline Neutrophils, median (IQR) & $8.7(5.5,13.5)$ & $4.3(2.9,6)$ & $<0.001$ & $\mathrm{n} / \mathrm{a}$ & $155.9(41.1,261.5)$ \\
\hline CRP, median (IQR) & $190.9(99.7,290)$ & $65(19.4,135)$ & $<0.001$ & $\mathrm{n} / \mathrm{a}$ & $95.51(52.17,756.67)$ \\
\hline IL-6, median (IQR) & $92.76(21.75,246.55)$ & 73.89 (30.9, 126.39) & 0.017 & $12.50(3.06,35.77)$ & $95.51(52.17,756.67)$ \\
\hline
\end{tabular}

$\Psi$ The sign rank test was used for continuous values and McNemar's test for categorical values.

- Other demographic details were not collected for this cohort.

The SARS-CoV-2 positive patients were age and gender matched with patients from the SAB and NIC cohorts.

TABLE 4B | Association with ICU admission (logistic regression separately for SARS-CoV-2 positive patients and SAB).

\begin{tabular}{|c|c|c|c|c|}
\hline & \multicolumn{2}{|c|}{ SARS-CoV-2 positive } & \multicolumn{2}{|c|}{ SAB } \\
\hline & $\begin{array}{c}\text { OR } \\
(95 \% \mathrm{Cl})\end{array}$ & $p$-value & $\begin{array}{c}\text { OR } \\
(95 \% \mathrm{Cl})\end{array}$ & p-value \\
\hline Female vs male & $0.55(0.16,1.91)$ & 0.349 & $2.81(0.80,9.92)$ & 0.108 \\
\hline Age (increase of 1 year) & $1.02(0.98,1.05)$ & 0.364 & $1.01(0.97,1.04)$ & 0.625 \\
\hline $\mathrm{CCl}$, median (IQR) & $0.90(0.59,1.39)$ & 0.641 & $0.93(0.68,1.27)$ & 0.652 \\
\hline WCC, median (IQR) & $0.99(0.92,1.07)$ & 0.879 & $0.96(0.861 .7)$ & 0.437 \\
\hline Neutrophils, median (IQR) & $1.15(0.94,1.41)$ & 0.163 & $0.96(0.86,1.08)$ & 0.505 \\
\hline $\begin{array}{l}\text { CRP (increase for } 10 \text { units), } \\
\text { median (IQR) }\end{array}$ & $1.13(1.03,1.23)$ & 0.008 & $1.06(1.00,1.12)$ & 0.034 \\
\hline IL-6 (increase for 10 units), median (IQR) & $0.95(0.87,1.03)$ & 0.232 & $1.07(1.02,1.12)$ & 0.01 \\
\hline
\end{tabular}

CCI, Charlson comorbidity index; CRP, C-reactive protein; ICU, intensive care unit; IL-6, interleukin-6; NIC, non-infectious conditions; SAB, Staphylococcus aureus bacteraemia; SARSCoV-2, severe acute respiratory syndrome coronavirus 2; SCOVID, suspected COVID-19; n/a, non-available data; WCC, white cell count.

TABLE 5 | Association of increased CRP and IL-6 values with outcome of severe disease (ICU admission, oxygen therapy or composite of both).

\begin{tabular}{|c|c|c|c|c|c|c|}
\hline & \multirow[t]{2}{*}{$\mathbf{N}$ total } & \multirow[t]{2}{*}{$\mathbf{N}$ with event } & \multicolumn{2}{|c|}{ Unadjusted } & \multicolumn{2}{|c|}{ Adjusted* } \\
\hline & & & SHR (95\% CI) & p-value & SHR (95\% Cl) & $p$-value \\
\hline \multicolumn{7}{|l|}{ ICU admission } \\
\hline IL-6 at baseline & 47 & 9 & $0.91(0.81,1.03)$ & 0.144 & $1.01(0.96,1.08)$ & 0.649 \\
\hline $\mathrm{IL}-6 \wedge^{\wedge}$ & 47 & 9 & $1.02(0.97,1.07)$ & 0.360 & $0.86(0.73,1.01)$ & 0.064 \\
\hline CRP at baseline & 54 & 14 & $1.10(1.05,1.14)$ & $<0.001$ & $1.05(0.96,1.15)$ & 0.249 \\
\hline $\mathrm{CRP}^{\wedge}$ & 54 & 14 & $1.09(1.05,1.14)$ & $<0.001$ & $1.06(0.98,1.14)$ & 0.149 \\
\hline \multicolumn{7}{|l|}{ Oxygen therapy } \\
\hline IL-6 at baseline & 51 & 21 & $1.00(0.97,1.03)$ & 0.960 & $1.00(0.96,1.04)$ & 0.973 \\
\hline$\| L-6 \wedge$ & 51 & 21 & $1.02(0.99,1.05)$ & 0.270 & $1.03(0.99,1.06)$ & 0.117 \\
\hline CRP at baseline & 54 & 24 & $1.08(1.04,1.12)$ & $<0.001$ & $1.05(1.00,1.10)$ & 0.047 \\
\hline $\mathrm{CRP}^{\wedge}$ & 54 & 24 & $1.07(1.03,1.11)$ & $<0.001$ & $1.05(1.01,1.10)$ & 0.013 \\
\hline \multicolumn{7}{|l|}{ Composite outcome } \\
\hline IL-6 at baseline & 46 & 18 & $1.00(0.97,1.04)$ & 0.807 & $0.99(0.94,1.05)$ & 0.795 \\
\hline $\mathrm{IL}-6^{\wedge}$ & 46 & 18 & $1.02(0.99,1.06)$ & 0.136 & $1.02(0.97,1.07)$ & 0.448 \\
\hline CRP at baseline & 54 & 26 & $1.09(1.05,1.14)$ & $<0.001$ & $1.06(1.01,1.11)$ & 0.020 \\
\hline $\mathrm{CRP}^{\wedge}$ & 54 & 26 & $1.09(1.05,1.13)$ & $<0.001$ & $1.05(1.01,1.10)$ & 0.025 \\
\hline
\end{tabular}

CRP, C-reactive protein; ICU, intensive care unit; Interleukin-6 (IL-6); SHR, sub-hazard ratio.

${ }^{*}$ Adjusted for respiratory rate (RR) (ICU admission), RR and SpO2 (oxygen therapy) and RR, SpO2 and age (composite outcome).

$\wedge$ Entered as a time-varying covariate.

Significant unadjusted values are shown in bold font.

frequently used in the clinical setting as a screening marker of infection and/or inflammation (12).

Although there are reports in the literature that an increase in IL-6 can correlate with disease severity in COVID-19 $(4,13)$, our study of a prospective SARS-CoV-2 cohort did not find that IL-6 levels offer a clinical utility for prediction of disease severity. We noted a stronger association between simple laboratory parameters (i.e. CRP) and bedside observations (i.e. $\mathrm{SpO}_{2}$ and respiratory rate) 
with disease severity, over IL-6. In our cohort, IL-6, CRP, ferritin and $\mathrm{LDH}$ were raised at hospital admission while lymphocytes were reduced in line with previous reports (14-16). Further, in another clinical prospective study on longitudinal immune profiling with a median of two time points of peripheral blood collection, the authors indicated an association between serum IL- 6 at the time of hospital admission and the severity of COVID-19, defined based on the degree of respiratory failure (16). Similar, in a larger retrospective longitudinal study $(\mathrm{N}=317)$, the authors showed the same pattern of increased inflammatory markers within the initial 24 hours after admission as described in our study and previously described study and correlation with disease severity for IL-6 more than $50 \mathrm{pg} / \mathrm{ml}$ on multivariable logistic regression (17). Vultaggio et al. (2020) found that in a cohort of 208 patients, 63 presenting clinical deterioration (defined as oxygen therapy, ICU admission, and death), IL-6 and CRP were predictors of negative outcomes in the first 3 days after hospital admission (18). In another study, maximal IL-6 (>80 pg/mL) and CRP (>97 mg/L) levels before intubation showed the strongest association with the need for mechanical ventilation in a cohort of COVID-19 hospitalized patients (19). These findings along with the results from our study support the use of CRP, a routinely available test, as a reliable predictor of clinical outcome in SARS-CoV-2 positive patients. Whilst, in our cohort, ferritin, D-dimer and LDH were not useful to monitor response to COVID therapies but, as discussed, were a marker of acute disease.

A limitation of the study in predicting severity of COVID-19 was the small sample size available in a low prevalence setting as well as the absence of confounders for most of the control groups (especially NIC). Our unique perspective of comparing IL-6 values among patients with other diseases, in particular those with an associated CS phenotype, provides insight into the underlying pathophysiology of SARS-CoV-2. CS is a somewhat controversial disease state with hyper-cytokinemia including IL-6 as key features (20). In a recent study from the Netherlands, the authors showed that IL- 6 values were lower in patients with COVID-19 and acute respiratory distress syndrome (ARDS) when compared to patients with septic shock with ARDS or septic shock without ARDS (21). This was supported by our research, illustrating lower IL-6 values in the SARS-CoV-2 positive patients compared to patients with $\mathrm{SAB}$. The idea that CS is not a prominent feature of severe COVID-19 as previously thought is growing in popularity and is supported by our study. Further examination of novel biomarkers in COVID-19 is required. In the interim, the use of routine tools such as CRP and bedside vital signs may offer the most reliability for clinical prediction.

\section{CONCLUSIONS}

- IL-6 levels in COVID-19 are elevated early in disease, although lower compared to other cytokine storm states.

- IL-6 levels follow the response to novel COVID-19 therapies, however do not offer a clinical advantage over C-reactive protein and bedside observations in predicting severe disease.

\section{DATA AVAILABILITY STATEMENT}

The original contributions presented in the study are included in the article/Supplementary Material. Further inquiries can be directed to the corresponding author.

\section{ETHICS STATEMENT}

The study was approved by the local human research ethics committee (Ref HREC/63201/Austin-20). All patients, their legal representatives or their next of kin provided oral rather than written consent for this study due to COVID-19 restrictions.

\section{AUTHOR CONTRIBUTIONS}

$\mathrm{AC}$ and FJ did the literature review and wrote the manuscript draft. AC and EM were responsible for the laboratory manipulations and data analysis. SV was the statistician responsible for this project. OS, CG, and GD were responsible for patient recruitment, sample follow-up and database entry. $\mathrm{NH}$ and JT proposed the study design and manuscript structure. All authors reviewed the current manuscript for important scientific content and made significant contribution to the different sections. All authors contributed to the article and approved the submitted version.

\section{FUNDING}

This study was supported by unrestricted funding from Austin Health Fundraising. JT was supported by the Austin Medical Research Foundation (AMRF) and by a National Health and Medical Research Council (NHMRC) postgraduate scholarship (GNT 1139902) and Royal Australian College.of Physicians Research Establishment Fellowship.

\section{ACKNOWLEDGMENTS}

We thank our research assistant, Mona Saade, and clinical nurse specialists, Wendy Stevenson and Kerryn McInnes for their assistance in sample coordination.

\section{SUPPLEMENTARY MATERIAL}

The Supplementary Material for this article can be found online at: https://www.frontiersin.org/articles/10.3389/fimmu.2021. 646095/full\#supplementary-material 


\section{REFERENCES}

1. Ruan Q, Yang K, Wang W, Jiang L, Song J. Clinical predictors of mortality due to COVID-19 based on an analysis of data of 150 patients from Wuhan, China. Intensive Care Med (2020) 46(5):846-8. doi: 10.1007/s00134-02005991-x

2. Chen G, et al. Clinical and immunologic features in severe and moderate Coronavirus Disease 2019. J Clin Invest (2020). doi: 10.1101/ 2020.02.16.20023903

3. Laguna-Goya R, Utrero-Rico A, Talayero P, Lasa-Lazaro M, RamirezFernandez A, Naranjo L, et al. IL-6-based mortality risk model for hospitalized patients with COVID-19. J Allergy Clin Immunol (2020) 146 (4):799-807 e799. doi: 10.1016/j.jaci.2020.07.009

4. Copaescu A, Smibert O, Gibson A, Phillips EJ, Trubiano JA. The role of IL-6 and other mediators in the cytokine storm associated with SARS-CoV-2 infection. J Allergy Clin Immunol (2020) 146(3):518-34.e1. doi: 10.1016/ j.jaci.2020.07.001

5. National Institutes of Health (NIH). Management of Persons with COVID-19 National Institutes of Health (NIH). (2020). Available at: https://www. covid19treatmentguidelines.nih.gov/overview/management-of-covid-19/, [cited $20207 / 10$ ].

6. Holmes NE, Robinson JO, van Hal SJ, Munckhof WJ, Athan E, Korman TM, et al. Morbidity from in-hospital complications is greater than treatment failure in patients with Staphylococcus aureus bacteraemia. BMC Infect Dis (2018) 18(1):107. doi: 10.1186/s12879-018-3011-2

7. Holmes N, Lemmens N, Robinson JO, van Hal S, Munckhof W, Athan E, et al. [P096] ANTI-STAPHYLOCOCCAL ANTIBODY PROFILE IN AUSTRALIAN PATIENTS WITH Staphylococcus aureus BACTERAEMIA. In: International Symposium on Staphylococci and Staphylococcal Infections. Copenhagen, Denmark (2018). Available at: https://isssi2018.org/fileadmin/ user_upload/ISSI2018/ISSSI2018_programme_abstract_book_web5.pdf

8. elisatkit.com. (2017). Product datasheet - Human IL-6 ELISA Kit. [cited 2020 6/10]. Available at: https://elisakit.com/wp-content/uploads/2018/06/0012Hu-IL-6-.pdf.

9. Trubiano A, Vogrin S, Smibert O, Marhoon N, Alexander A, Chua K, et al. COVID-MATCH65 - A prospectively derived clinical decision rule for severe acute respiratory syndrome coronavirus 2. medRxiv (2020). doi: 10.1371/ journal.pone.0243414

10. Tanaka T, Kishimoto T. The biology and medical implications of interleukin-6. Cancer Immunol Res (2014) 2(4):288-94. doi: 10.1158/2326-6066.CIR-14-0022

11. Uciechowski P, Dempke WCM. Interleukin-6: A Masterplayer in the Cytokine Network. Oncology (2020) 98(3):131-7. doi: 10.1159/000505099

12. Faix JD. Biomarkers of sepsis. Crit Rev Clin Lab Sci (2013) 50(1):23-36. doi: $10.3109 / 10408363.2013 .764490$
13. Del Valle DM, Kim-Schulze S, Huang HH, Beckmann ND, Nirenberg S, Wang $\mathrm{B}$, et al. An inflammatory cytokine signature predicts COVID-19 severity and survival. Nat Med (2020) 26(10):1636-43. doi: 10.1038/s41591-020-1051-9

14. Henry BM, de Oliveira MHS, Benoit S, Plebani M, Lippi G. Hematologic, biochemical and immune biomarker abnormalities associated with severe illness and mortality in coronavirus disease 2019 (COVID-19): a meta-analysis. Clin Chem Lab Med (2020) 58(7):1021-8. doi: 10.1515/cclm-2020-0369

15. Terpos E, Ntanasis-Stathopoulos I, Elalamy I, Kastritis E, Sergentanis TN, Politou M, et al. Hematological findings and complications of COVID-19. Am J Hematol (2020) 95(7):834-47. doi: 10.1002/ajh.25829

16. Mann ER, Menon M, Knight SB, Konkel JE, Jagger C, Shaw TN, et al. Longitudinal immune profiling reveals key myeloid signatures associated with COVID-19. Sci Immunol (2020) 5(51). doi: 10.1126/sciimmunol.abd6197

17. Zeng Z, Yu H, Chen H, Qi W, Chen L, Chen G, et al. Longitudinal changes of inflammatory parameters and their correlation with disease severity and outcomes in patients with COVID-19 from Wuhan, China. Crit Care (2020) 24(1):525. doi: 10.1186/s13054-020-03255-0

18. Vultaggio A, Vivarelli E, Virgili G, Lucenteforte E, Bartoloni A, Nozzoli C, et al. Prompt Predicting of Early Clinical Deterioration of Moderate-to-Severe COVID-19 Patients: Usefulness of a Combined Score Using IL-6 in a Preliminary Study. J Allergy Clin Immunol Pract (2020) 8(8):2575-81.e2. doi: $10.1016 /$ j.jaip. 2020.06 .013

19. Herold T, Jurinovic V, Arnreich C, Hellmuth J, von Bergwelt-Baildon M, Klein $\mathrm{M}$, et al. Level of IL-6 predicts respiratory failure in hospitalized symptomatic COVID-19 patients. medRxiv (2020). doi: 10.1101/ 2020.04.01.20047381

20. Tisoncik JR, Korth MJ, Simmons CP, Farrar J, Martin TR, Katze M. Into the eye of the cytokine storm. Microbiol Mol Biol Rev (2012) 76(1):16-32. doi: 10.1128/MMBR.05015-11

21. Kox M, Waalders NJB, Kooistra EJ, Gerretsen J, Pickkers P. Cytokine Levels in Critically Ill Patients With COVID-19 and Other Conditions. JAMA (2020). doi: $10.1001 /$ jama.2020.17052

Conflict of Interest: The authors declare that the research was conducted in the absence of any commercial or financial relationships that could be construed as a potential conflict of interest.

Copyright @ 2021 Copaescu, James, Mouhtouris, Vogrin, Smibert, Gordon, Drewett, Holmes and Trubiano. This is an open-access article distributed under the terms of the Creative Commons Attribution License (CC BY). The use, distribution or reproduction in other forums is permitted, provided the original author(s) and the copyright owner(s) are credited and that the original publication in this journal is cited, in accordance with accepted academic practice. No use, distribution or reproduction is permitted which does not comply with these terms. 